\title{
Study of Adaptive Beam Forming with Direction Selector of Target Sound by Hearing Aid User
}

\author{
Tsubasa Noguchi ${ }^{\mathrm{a},}{ }^{*}$, Mitsunori Mizumachi $^{\mathrm{a}}$ Yoshihisa Nakatoh $^{\mathrm{a}}$ \\ ${ }^{a}$ Kyushu Institute of Technology, 1-1 Sensui-cho, Tobata-ku, Kitakyushu 804-8550, Japan \\ *Corresponding Author: o595401y@mail.kyutech.jp
}

\begin{abstract}
The main purpose of this study is to examine the beam forming of the hearing aid for hearing aid wearers (users) to be able to select a target sound direction. In Section2, we will show system flow, the UI, and principles of adaptive beamf orming. In Section 3, we evaluate the relationship between sound source direction and user input by using UI. In Section 4, we discuss the adaptive beam forming method on the basis of the results of Section3.
\end{abstract}

Keywords: Hearing Aid, Beam Forming,

\section{Introduction}

It is inferred to have 19.44 million hearing-impaired people in Japan according to Japan Industry Association Hearing Aids. This is about $15 \%$ of the total population of Japan. Hearing aid plays an important role of communication for the hearing impaired. Hearing aid performs noise reduction by using the characteristics of sound and directionality. Noise reduction using direction exhibits high performance by assuming target sound direction to be front of user. However, in the cases that a target sound is not in front, a hearing aid can't sufficiently exhibit performance. Therefore, it is impossible for user to hear like people who can use cocktail party effect. In this paper, we consider a hearing aid system that can improve the intelligibility even if a target sound is in any direction by incorporating the direction that inputted by users.

\section{Noise Reduction that Incorporates a Direction that Inputted by User}

The hearing aid system of this study is aimed at improving the intelligibility for arbitrary directions by incorporating of direction input by users to adaptive beam forming conventional. Therefore, we made a hearing aid system using a smart phone as a direction input device. In this chapter, we explain the flowchart of this hearing aid system.

\subsection{Flow of the Hearing Aid System that We Propose}

Fig. 1 is the flowchart of the hearing aid system that we propose. The input is a tap from the smartphone and acoustic signals input from a microphone. The acoustic signal input is a mixed sound of the noise and target sound. In order to extract the target sound from the mixed sound, the proposed system uses adaptive beam forming that is one of the array signal processing. Adaptive beam forming of a conventional hearing aid is a mechanism that attenuating the sound coming from other than the front by assuming that the target sound is in front. The system is able to discern the sound coming from any direction by assuming the direction of target sound to the direction input on the smart phone.

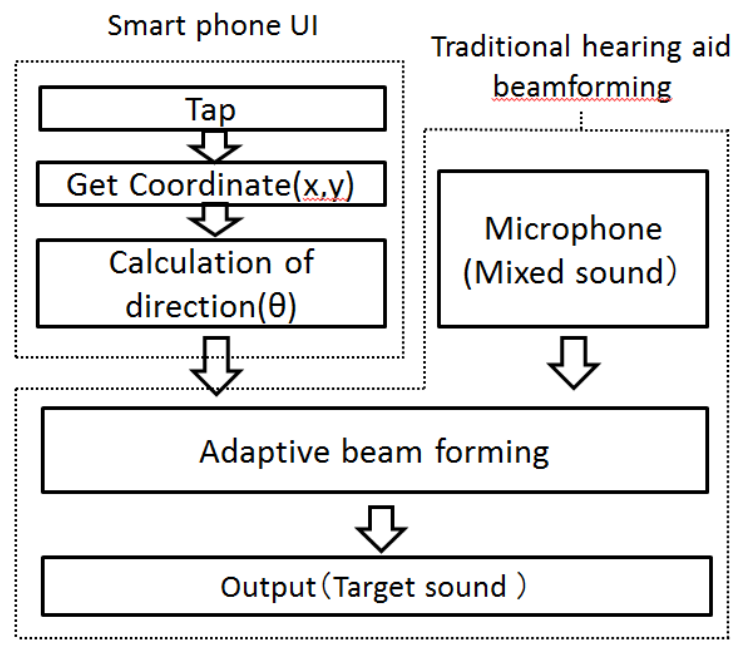

Fig. 1 Flowchart 


\subsection{The UI of Smart Phone as a Direction Input Device}

When the user inputs a direction using the smartphone, we must make the UI which can input direction intuitively. Therefore, we have created the UI. Fig. 2 is its UI. Fig. 2 represents the space around with a focus on user. In Fig. 2 (i), it is set to $0^{\circ}$ front direction, $90^{\circ}$ right direction, $-90^{\circ}$ left direction, $180^{\circ}$ behind direction. Therefore, the users can predict the direction of a target sound from themselves. The user inputs the direction of target sound by moving the mark. The mark moves to the position that was tapped by tap input, finely adjusted by dragging.

\subsection{The Beam Forming Incorporating the UI Input}

Beam forming, which is one of the noise reduction techniques, is a method of utilizing an array signal processing using a plurality of microphones. We show principle of beam forming Fig. 3, an example of a directional beam forming by in Fig. 4. Addition type does not provide sufficient performance in general, when scale of the array is not large. However, subtractive is able to exhibit a satisfactory performance in a small array. Therefore, adaptive beam forming that combines subtractive and addition type is used in a small array, such as a hearing aid.

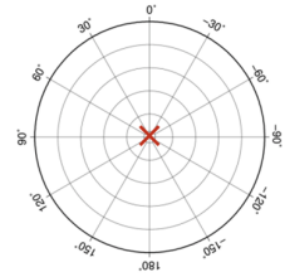

(i) Scale included

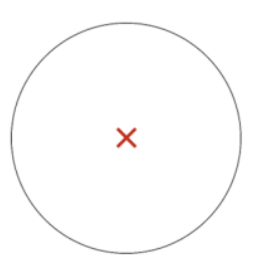

(ii) Without scale
Fig. 2 UI which can input a direction of target sound

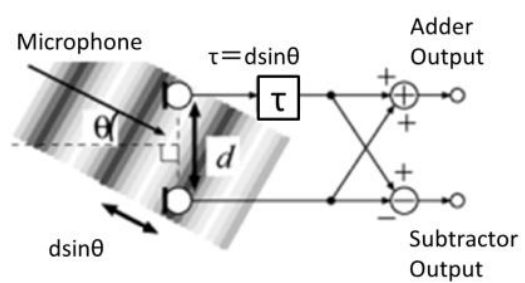

Fig. 3 Principle of beam forming (reprinted from [5])

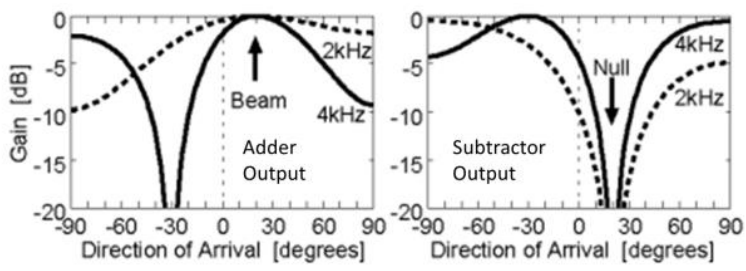

Fig. 4 Example of a directional beam forming (reprinted from [5])
Fig. 5 is an example of a directionality obtained by the adaptive beam former. Adaptive beam forming to exhibit high noise rejection by forming target sound direction of beam and forming noise direction of null as in Fig. 5. Because the noise and the target sound change in real situation in the operating environment of the hearing aid, it is necessary to estimate each sound source direction, However if it is not able to correctly estimate target sound direction, and it degrade the target sound by the adaptive operation there is a possibility that the put away. Therefore, if it is attenuated estimated by adaptive signal processing only noise direction is fixed to front direction of the target sound direction is large in a conventional hearing aid. Griffiths-Jim beam former [9] is well known as a typical example of the adaptive beam forming such.

Adaptive beam forming of the hearing aid is obtained by applying the Griffiths-Jim beam former. [10] Fig. 6 is a principle that is set to input direction of the UI, target sound direction. in the adaptive beam forming of the hearing aid. By using this method, it is not necessary to direct the face in the direction of the target sound, the user is able to discern the sound on the front outside. The problem with this system is the error between the target sound with the original direction and the direction input using a smart phone. Small estimation error of the sound source direction is a significant impact on performance in the beam forming. Therefore, how to reduce the negative effects of errors is the subject of the proposed method.

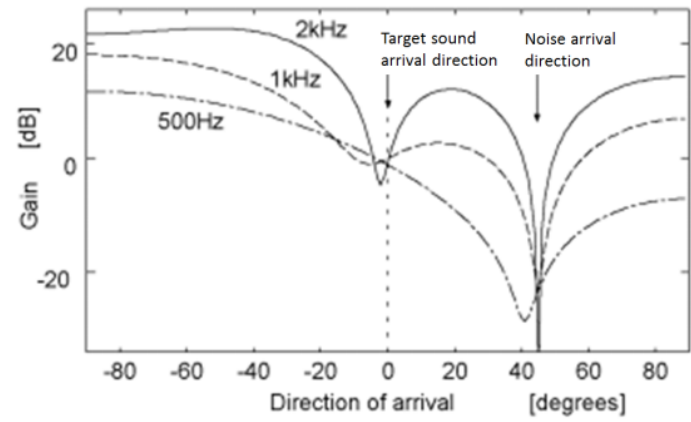

Fig. 5 Example of a directionality obtained by the adaptive beam former ${ }^{\text {(reprinted from [5] }}$

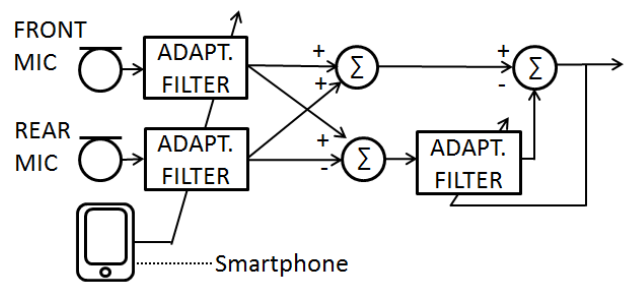

Fig. 6 Principle using the UI input of smart phone 


\section{Accuracy to Input the Target Sound Direction using a Smart Phone}

How to reduce the effect of error is an issue adaptive beam forming proposed in Section 2.2. Therefore, we have experiments on the accuracy of the input sound source direction by UI.

\subsection{Experimental Methodology}

Subjects input using the UI which is shown in Fig. 2 the direction of the loudspeaker becomes a voice in the experiment. Loudspeaker was placed eight around the subject, the sound is played in random from one of them. Where the experiment was performed is a soundproof chamber. Because it is assumed that the actual operating environment, without changing the orientation of the face, it is determined by visual and auditory position of the speaker of $20^{\circ} \sim 80^{\circ}$ direction, the position of the speaker of $100^{\circ} \sim 160^{\circ}$ direction is determined by the only hearing were. I was in private the position of the sound source to the subject. Adult male subjects 17 people, 14 of whom use the UI (i), 3 people were used UI (ii). Sound source that was used men read speech that is included sound library [4], the measurement was carried out a total of 80 times in the 10 times each direction. Fig. 7 is a figure showing the experimental environment.

\subsection{Experimental Result}

A common feature in 14 people 17 people appeared in the experimental results. In addition, different characteristics in subjects using the UI of Fig. 2 (ii) and subject using the UI of Fig. 2 (i) was also observed. Fig8 is a plot of one by one measurement result. SubjectAB using the UI there scale, subjectCD using a scale no UI. Fig. 9 is a figure that shows the mean and standard deviation of the results of Fig. 8.

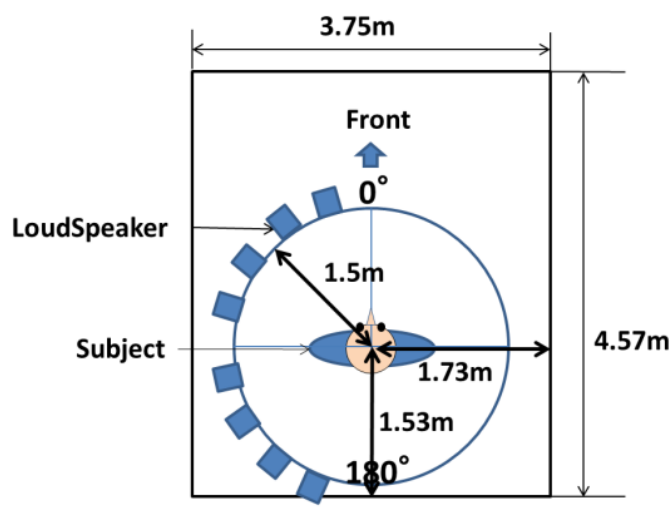

Fig. 7 Experimental environment

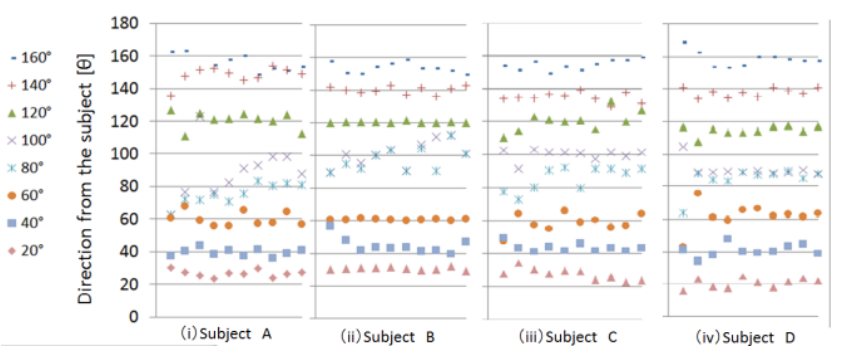

Fig. 8 Plot of one of the measurement results

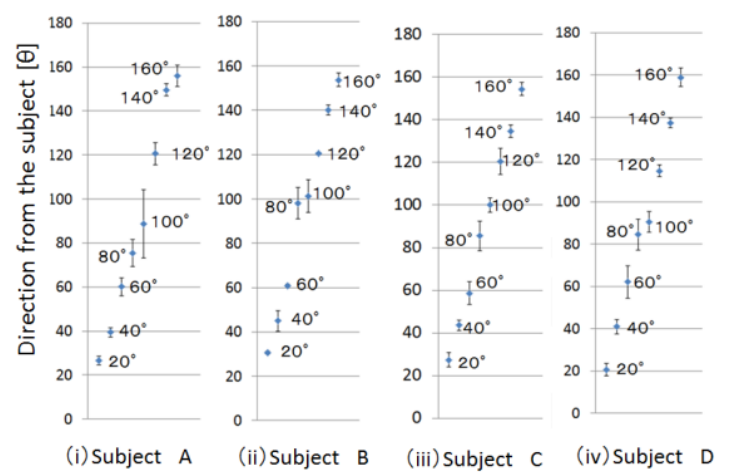

Fig. 9 Mean and standard deviation of the measured values

\subsection{Discussion of the Experimental Results}

\subsubsection{Differences in Accuracy in Each Direction}

A common feature is the difference of accuracy in each direction on 14 subjects. Error is within $\pm 10^{\circ}$ any subject in the direction $20^{\circ}, 40^{\circ}, 60^{\circ}$. In addition there is a tendency for the standard deviation is reduced closer to the front. Error of mean and standard deviation is large the result of Fig9,10 the direction $80^{\circ}, 100^{\circ}$. It is found that it is difficult therefore, to distinguish the sound or the sound either. In the $120^{\circ}, 140^{\circ}, 160^{\circ}$ direction, 14 people 17 people during was within $\pm 10^{\circ}$ the error of the mean. However, the standard deviation was the result is greater as compared with the direction $20^{\circ}, 40^{\circ}, 60^{\circ}$. Standard deviation is differently for each subjects, However the subject standard deviation was the result of 5 or more, was nine people at $120^{\circ}$, and three people at $140^{\circ}$, one people at $160^{\circ}$. From this result, it was found that standard deviation is smaller as it approaches behind when viewed in all subjects.

\subsection{Effects of UI}

Subject A was greater the error of the mean direction 20,140. The above causes can be inferred to be because subject A have already aware of the separator screen of UU direction in the UI screen. Mean of eight people in 14 of the subjects was influenced by the UI as the subject A. 3 subjects using the UI in Fig. 2 (ii) has resulted 
Table. 1 Input direction and original direction

\begin{tabular}{|c|c|c|}
\hline \multicolumn{2}{|c|}{} & $\begin{array}{c}\text { Direction of desired } \\
\text { source of sound }(\boldsymbol{\theta 0})\end{array}$ \\
\hline \multirow{3}{*}{$\begin{array}{c}\text { Inputted } \\
\text { direction } \\
\text { by the user }(\boldsymbol{\theta})\end{array}$} & $\mathbf{0} \leqq \boldsymbol{\theta} \leqq \mathbf{8 0}$ & $\theta-10^{\circ} \leqq \theta \mathrm{o} \leqq \theta+10^{\circ}$ \\
\cline { 2 - 3 } & $\mathbf{8 0} \leqq \boldsymbol{\theta} \leqq \mathbf{9 0}$ & $\theta-10^{\circ} \leqq \theta \mathrm{o} \leqq \theta+20^{\circ}$ \\
\cline { 2 - 3 } & $\mathbf{1 0 0 \leqq} \leqq \mathbf{\theta 0 0}$ & $\theta-20^{\circ} \leqq \theta \mathrm{0} \leqq \theta+10^{\circ}$ \\
\hline
\end{tabular}

$\theta$ : Input direction, $\theta_{\mathrm{o}}$ : original direction

standard deviation is high. However, error of the mean was small results compared with subjects with the UI of Fig. 2 (ii).

\subsubsection{Summary of experimental results}

The results of this experiment, the error due to the input of the sound source direction using a smart phone has been found that it is necessary to consider the error range according to each direction. Based on a consideration of Section 3.3.1, is shown in Table. 1. the correspondence between the original direction and the input direction.

\section{Study of Adaptive Beam Forming using the UI Input}

Order to reduce the influence of the error of the target sound direction, we propose a new method for varying the beam width of the target sound direction in accordance with the input accuracy in each direction.

\subsection{Consideration of Appropriate Beam width in Each Direction}

Width of the beam (range not to degrade the signal) should include a target sound the original direction of Table1. According to the literature [10], the performance of the array of Fig. 6 is about $\pm 10^{\circ}$ beam width. If it is assumed that can form a beam in the beam width in all directions, the beam at $80^{\circ} \sim 100^{\circ}$ direction must be changed to a width that takes into account the Table. 1.

\subsection{Adaptive Beam Forming using Directional Target Sound Inputted}

Fig. 10 is a beam forming method proposed. Usually, Griffiths-Jim beam former is to form a main beam one target sound direction. Proposed method includes a sub-beam former to $\pm 90^{\circ}$ direction separately from the main beam. If $80 \sim 100{ }^{\circ}$ direction is input, changes the width of the beam by adding together the main beam by forming a sub-beams. In this way, even if there is an error in the target sound direction inputted by the smartphone, adaptive beam forming process that does not degrade the target sound is possible.

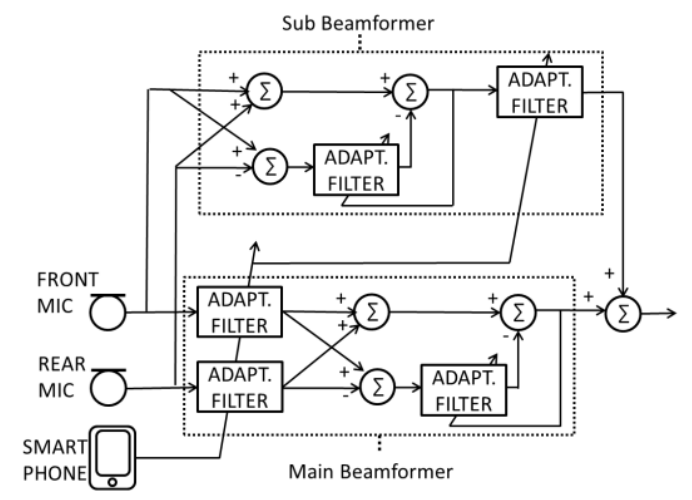

Fig. 10 Adaptive beam former of the proposed method

\section{Conclusion}

In this study, we propose a noise reduction system of hearing aids that incorporates the input intuitive smartphone, so measure the accuracy of the input of the UI is a problem by experiment. In addition, we have proposed an adaptive beam forming method that takes into account the accuracy of the UI input which has been found from the experimental results. We plan to evaluate the performance of the adaptive beam former proposed in the future.

\section{References}

[1] Japan Hearing Instruments Manufacturers Association "Hearing aid systems in assistive technology" (2002)

[2] Tatsuo Nakagawa, Masayuki Sato, Masahiro Takahashi "Sound localization under amplification" IEICE technical report. SP, voice 113(76), 61-64, 2013-06-06

[3] Armstrong, S. "Hearing aid algorithms." American Audiology Society 2006 meeting, Scottsdale, AZ, March 7, 2006

[4] Yutaka Kaneda, Hutoshi Asano, Kenichi Huruno, Yoshinobu Kazikawa,Osamu Hoshuyama "Forest of knowledge-Acoustic signal processing" Chapter 2-1 Beamforming (2012)

[5] L.J. Griffiths and C.W. Jim "An Alternative Approach to Linear Constrained Adaptive Beamforming,"IEEE Trans. AP, vol.AP-30,no.1, pp.27-34, Jan.1982

[6] Ma,J.-B,Wouters,J.,\& Moonen,M. "Noise reduction resukts of an adaptive filtering technique for dual-microphone behind-the-ear hearing aids." Ear and Hearing, 25, 215-229. (2004). 